\title{
Investigating the Domestic Layers Adaptation
}

\section{During Pandemic}

Afifah Karimah, Kristanti Dewi Paramita $\quad \begin{aligned} & \text { Universitas Indonesia } \\ & \text { Indonesia }\end{aligned}$

\begin{abstract}
This paper expands the theoretical understanding of building layers proposed by Brand (1995) by investigating changes in the domestic environment during the COVID-19 pandemic. Brand's layer framework breaks a built environment into "shearing layers" to examine its adaptation processes. This paper argues that ways of managing the risk of virus transmission in the built environment redefine the understanding of these layers. This paper takes the perspective of interiority to address these layers as instruments with the spatial qualities required of a resilient domestic environment. The study unpacks the theory of Brand's layer framework, proposing the principles by which layers adapt to protect the domestic environment during the COVID-19 pandemic. It then offers readings on the occurrence of change in the domestic environment in which such adaptation principles are performed. Such occurrences consist of intensifying layer changes to assist intense uses, merge between layers to assist movements, the construction of new layer forms, and reconfiguration of multiple layers for a prolonged change. Apart from redefining the very understanding of layers, this paper addresses how spatial change is not driven only by physical deterioration, but also by the performative creation of scenarios to protect the domestic environment during the pandemic.
\end{abstract}

Keywords: layers framework, change, performance, domestic environment, COVID-19

Correspondence Address: Afifah Karimah, Department of Architecture, Faculty of Engineering, Universitas Indonesia, Kampus UI, Depok 16424, Indonesia. Email: afifahkar@ui.ac.id 


\section{Introduction}

Domestic environments continuously change and adapt during inhabitation. This paper seeks to better understand such change during the current COVID-19 pandemic. The paper bases its exploration on Brand's "shearing layers of change" proposition (Brand, 1995), which demonstrates how a built environment consists of six layers - the site, skin, structure, service, space plan, and stuff layers. Through examining changes in various buildings from the 1800 s to the 1990s, Brand argues against the view of buildings in modernist architecture as the eternal "crystalline" that can anticipate growth (Brand, 1995, p. 2). Instead, he suggests that building can be examined only as a whole by understanding its passage through time, and this examination can be done by separating building into layers. Apart from gaining insight into how buildings change, understanding buildings as layers is part of the growing discourse that aims to shift the perspective of architecture as singular, durable, and timeless (Handa, 2015; Till, 2013).

During the COVID-19 pandemic, restrictions on contact with the outer world have forced the layers of the domestic environment to adapt and take measures necessary to increase domestic resilience towards virus transmission. This paper proposes to re-read the understanding of layers using an interiority perspective that emphasises the formation of layers and the connections between, which construct the domestic environment's spatiality (Lens \& Van Cleempoel, 2015; Weinthal, 2011). This focus on performance and interaction between layers will potentially expand on Brand's shearing layers framework, creating a more holistic understanding of change in a domestic context.

This paper starts by revisiting Brand's (1995) notion of layers as a framework through which to see spatial change, highlighting each layer's definition and its overall significance for the wider discourse on architecture and interior. This paper then uses discussion of interiority to re-read the adaptation processes of layers in a domestic environment against the threat of COVID-19 transmission. The paper then proposes the principles of layers' adaptation, re-reading how adaptation produces change in a domestic environment towards a resilient interior. By doing so, the study expands the understanding of layers as a framework for seeing changes in the domestic environment. 


\section{Seeing the Built Environment Through Layers: Meanings and Significances}

Brand's theoretical proposition is an expansion of Frank Duffy's (1990) idea of a built environment as a set of four building layers, increasing Duffy's four layers to the six layers. They consist of: the site layer as the location where the building stands; the structure layer as the structural elements which support the building, then the skin layer as the exterior covering surface that deals with the weather; the service layer as the working guts of the building system (such as the electricity, sanitation, and the ventilation systems); the space plan layer as the layout of space defined by the built physical elements and openings; the stuff layer, comprising objects that are more likely always move around and got replaced more often (Figure 1). Brand (1995) argues that each layer has a different rate of change and adapts on its own; as each layer adapts, the built environment is continuously reinvented in response to the process of inhabitation.

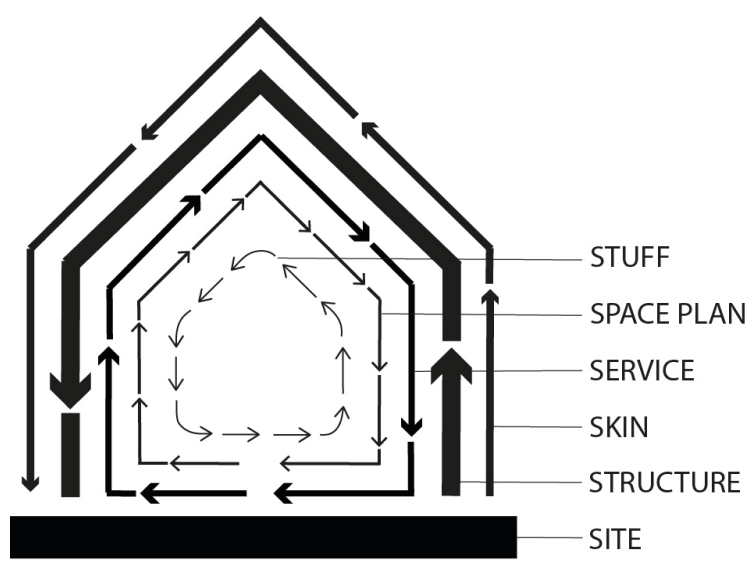

Figure 1

The shearing layers of change diagram by Brand (1995) (Image redrawn by authors)

Brand's proposition is important for allowing an understanding of changes in the built environment by breaking the environment into layers. His proposition recognises a variety of inhabitation conditions and forces (Alexander, 1979; de Certeau, 1984; Franck \& Lepori, 2000) that may drive different responses from parts of the built environment. Included among these conditions and forces are physical wear and tear, exposure to weather and climate conditions, technological advancement making previous systems or objects obsolete, or even moments of individual impulse or necessity (Adams \& Marlor, 2019; Brand, 1995; Marinic, 2018; Schneider \& Till, 2007; Till, 2013). The presence of these conditions and forces means that architecture is never fully completed or perfect; it is continuously, gradually adapting in a piecemeal way rather than transforming all at once (Alexander, 1975; Brand, 1995). 
While Brand's investigation examines how the layers are exposed and respond to the gradual threat of physical deterioration and obsolescence, this paper argues that the current COVID-19 pandemic has created a different threat that is driving change more rapidly. Faced with such rapid change, each layer does not exist and changes on its own, but interacts with one another, and collectively become active spatial instruments that produce or present a particular condition or arrangement of space. While Brand's shearing layers framework is applied primarily in discourse on responsive or sustainable architecture, this article points out that the understanding of layers as spatial instruments is particularly relevant to interior architecture discourse. Rather than regarding layers as building parts, such discourse uses the term layers to examine how the built environment intentionally confronts and reveals the temporal dynamics of an inhabitation (Attiwill, 2012; Marinic, 2018; Warakanyaka \& Yatmo, 2018).

Warakanyaka and Yatmo (2018b) have presented a related study investigating cracked facades, paints flakes, and mismatched tiles to demonstrate the stories of inhabitation through time, which become an important aspect of the interiority of a conservation building. Seeing a built environment in layers from an interiority perspective demonstrates how different layers produce different spatial articulations of environmental qualities that are collectively important for the space (Banham, 1984; Franck \& Lepori, 2000; Leatherbarrow, 2002; Marinic, 2018). The present study seeks to understand how the activity of layers generates spatial articulation to create a more resilient domestic space. The following sections explore these articulations and qualities further with particular attention to the domestic environment during the COVID-19 pandemic.

\section{Layers' Adaptation and Connections During a Pandemic}

Until now, respiratory and physical contact have been declared the two main routes of COVID-19 virus transmission (WHO, 2020). Both types of transmission are caused by the production of infectious droplets by people with COVID-19 and the distribution of those droplets to their surroundings through coughing, sneezing, physical touching, sharing food or talking (WHO, 2020). The infectious droplets might also stay on surfaces where the virus remains viable for a duration and creates infection risks (Dietz et al., 2020; van Doremalen et al., 2020; Wang et al., 2020; WHO, 2020).

An array of guidelines, requirements and health protocols for physical interaction have been provided by health practitioners 
and researchers (WHO, 2020). Some examples of these protective measures are frequent hand-washing, frequent disinfection of high-touch surfaces, keeping infectious objects outside the reach of others and incubating infectious objects at a certain degree of heat if possible (Bloomfield et al., 2012; Dietz et al., 2020; Duan et al., 2003; WHO, 2020; Yatmo et al., 2020).

These protective measures significantly alter the domestic environment by requiring it to accommodate new activities or the intensification of existing activities, in addition to complicating the procedure of doing the activities. Some new activities are working or studying from home and physical exercise that was previously done outside the home, while intensified activities include food preparation, or other activities that require family members to gather and share space.

This paper proposes some new principles of layers' adaptations to assist with such changes in the domestic environment. The first important principle of layers' adaptation is that each layer does not adapt solely on its own but might interact or combine with another layer. For example, studies have demonstrated that the sanitary and ventilation systems are significant in distributing potentially contaminated droplets through the water and airflow of a dwelling (Banham, 1984; Yatmo et al., 2018). However, a dwelling's spatial layout also directs the potentially contaminated airflow (Yatmo et al., 2018). This demonstrates that the service layer must work with other layers, such as the space plan layer.

Apart from interaction among themselves, the next principle highlights some layers might take on another form. This may occur when activities in the dwelling are done in a different form, such as online learning or online physical fitness classes. This technological advancement allows a safe connection to the outside world. The virtual interface acts as an expansion of the domestic environment to the outside world, creating a "virtual window" (Friedberg, 2006, p. 151). It is not only the instrument of seeing but also the instrument of adjustments (Leatherbarrow \& Mostafavi, 2002) by arranging the types of communications, targeted actors, and timing of the encounters. In this sense, the transformation of activities potentially demonstrates the alternative forms of layers.

The last principle highlights how positions of layers may go beyond Brand's configuration of layers from outside to inside. There might be an "interwoven" relation between inside and outside (Martinelli, 2019, p. 147), particularly on dealing with space limitations (Klasto, 2019) that produces the required qualities of the interiority. There is a potential that layers can be positioned differently within the 
domestic environment, for example, with the recommendation of placements of hand-washing facilities at the boundaries of home and before entering the neighbourhood (WHO, 2020).

With these new principles for layers' activity, Brand's diagram that visualises layers' configuration might change. Brand's diagram aims to depict the layers' different rates of change, their changing behaviour and the order of their positions through the thickness of lines and quantity of arrows. The first four, thicker lines represent layers that are more stable or that change very slowly: the site, structure, skin, and service layers. The last two, thinner lines represent more quickly changing layers: the space plan and stuff layers (Brand, 1995). All layers are placed in a certain order, from the outside to the inside of the built environment (Brand, 1995). Based on the principles offered in this section, the following section alters and further expands on these visualisations.

\section{Re-reading the Changing Layers during COVID-19 Pandemic: Adaptations and Configurations}

The previous sections have highlighted some adaptation principles of the layers of built environments during the pandemic; from the interaction between layers, to the creation of new forms of layer, and the order of the layers' positions. The following paragraphs aim to re-read and discuss how these principles apply to different layers' performance of specific spatial qualities to prevent virus transmission and construct a resilient, interior domestic environment that enables safe inhabitation during a pandemic. This discussion becomes the basis for visualising the new compositions of the layers' configuration are offered.

\section{Intensifying layer changes throughout the day based on an increased variation of uses}

With stay-at-home requirements, the domestic environment is used more intensely, for longer periods of time and by more people. Such unusual use drives the rearrangement of some layers to accommodate the various activities and dwellers. Components of the stuff layer, such as domestic objects and furniture, are used and rearranged intensively throughout the day. For example, sitting spaces in the living room can be used by multiple dwellers for eating, for school and work-from-home activities and for physical fitness exercise.

Space may need to be rearranged in certain ways, to create private workspaces for school at home, for example, and to remove them to 
make space for physical fitness exercise. In addition, additional stuff might be required, becoming part of the change, such as portable laptop stands or desk lamps required for video calls.

Apart from such rearrangements, some layers may also be used for longer periods than usual, such as longer and more frequent food preparation due to decreased eating-out events and additional family members staying at home. Longer use times also emphasise the service layer because of increased needs for water, electricity, and air conditioning systems. The diagram in Figure 2 demonstrates the intensification of the stuff, space plan, and service layers to accommodate longer and more frequent activities throughout the day. The diagram depicts how the site layer transcends to the inside of the dwelling and becomes more than the location where the dwelling is situated. The orange-coloured layer demonstrates how the layer moves around to manage the changes at home, while layers represented in black colour remain in their position, but are used more heavily. The grey-coloured layers are those that remain unchanged in terms of uses nor positions.

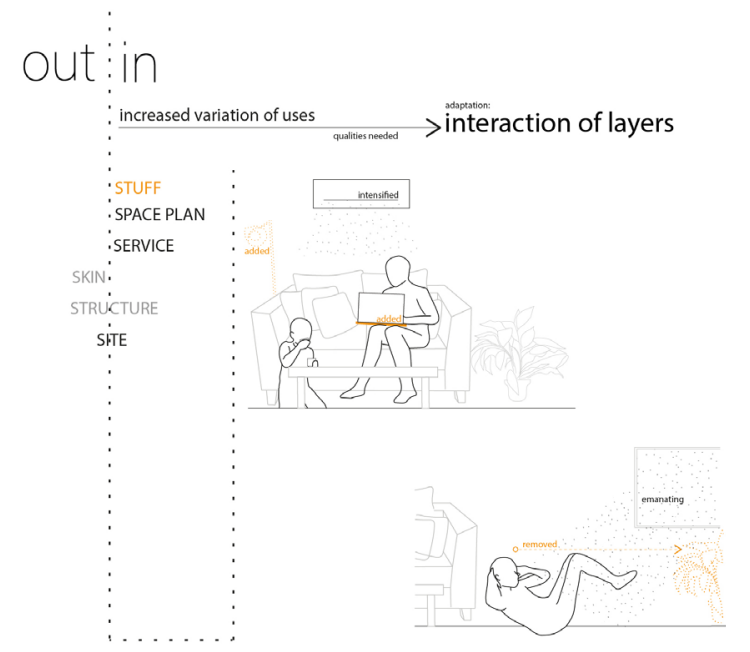

Figure 2

More intensified

usages or rearrangements of some layers (Image by authors)

Other than the rearrangement of stuff and the extended use of services, inhabitants' constant activity in the same built environment makes them more aware that the site layer is not simply the building's location, but also enables the qualities, such as sunlight and airflow, that support some of their activities. For example, dwellers may find the brightest space in the dwelling to occupy during a video call, or they may sunbathe by sitting on the terrace to boost their immune system. These qualities might be particularly in demand during the pandemic, creating an interaction between the stuff, space plan, and site layers. 


\section{Merging layers: Assisting movements of self and objects between inside and outside}

During the pandemic, some layers perform together and even merge. With this merging, some layers' positions are twisted from inside to outside and vice versa. Layers might merge to provide temporary protection and enable inhabitation within the changing context. The first layer to change its position is the stuff layer, which is usually placed inside but is shifted to the outside during the pandemic. An example would be the dropbox placed outside to contain the items ordered online, which have increased with the stay-at-home requirements. The dropbox is part of the stuff layer but is placed at the border the outside and the inside of the home. When a package arrives at the inside, it is placed in the dropbox for a period because infectious droplets might have become lodged on the package's surface along the journey. The dropbox acts as a temporary container at the edge of the domestic space. It can therefore be argued that, as objects transition from the outside to the inside of the domestic environment during a pandemic, the skin and stuff layer merge with each other, adapting to current events.

Figure 3

The process of merging some layers into one layer (Image by authors)
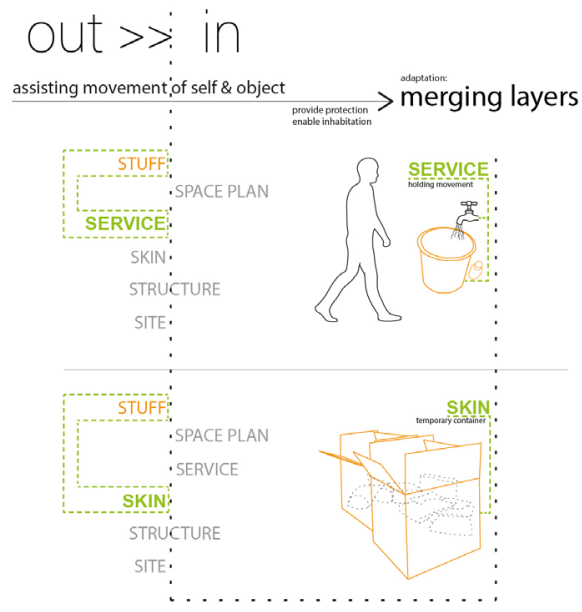

The diagram in Figure 3 demonstrates how some layers have moved outside the house or merged to another layer. The position of the stuff layer around the black line represents the layer as parts of the domestic space's boundary, merging the stuff layer with the skin and the service layer. The orange-coloured layer moves around the dwelling and then merges together as the green-coloured layer. Both layers are grouped together with enclosed green lines, indicating the interaction between them that drives the merging process. 
In addition to the stuff layer, the service layer merges with the skin layer to assist the entry process to the domestic space. An example of such a merge is placing a water bucket or an emergency water tap to help dwellers clean their hands before entering the domestic environment. The presence of a water source the dweller must engage with before moving inside the home demonstrates the merging of the service and skin layers to protect the domestic environment, impeding people from entering before they can be deemed safe from transmitting the virus.

\section{Constructing new forms of layers which drive further change}

This section draws attention to the new forms of layers, that are constructed by the incorporation of stuff or systems, that were not initially included as part of the spatial layers, but are now vital within the occupation of space. Such incorporation is driven by the new procedures and the use of new technology or tools within the domestic environment. These procedures and technologies create a new system of living and drives the establishment of an entirely new form of layer. Examples of these new forms of layer are the use of mask or body coverage applied in the process of moving in and out the dwelling, or the presence of virtual interface at devices that replaces offline interaction. It can be argued that the mask and the virtual interface demonstrate new forms of the skin layer.

The diagram in Figure 4 summarises the emergence of the layers' new forms and how they drive further change. The green-coloured layer indicates the one that has a new form, driven by new procedures and new technologies. The new form of layer may interact with other layers directly, which are coloured in orange. Some of these interacting layers might move around the dwelling while some remain, as indicated in the layer's position in relation to the black lines. The enclosing green lines group the different layers that are interrelated together.

In the process, these new forms of layer might generate relations to other layers, influencing further change. For instance, the use of masks may influence positions and distances between people and their objects, therefore influencing the space plan layer. Such change of space plan can be seen in the change of the domestic space to accommodate the presence of visiting guests (i.e repairmen,

commuting household assistants). During the presence of such occupants, masks and other covering tools (such as food coverage, cushion coverage, and so on) are applied to the stuff and arranged in a layout that provides protective coverage for the rest of family 
Figure 4

The form of

new layers

constructed by multiple layers acting together (Image by authors)

members. In this sense, the use of masks and other covering tools as a skin layer generates relation to the stuff and space plan layer in driving change to the domestic environment.

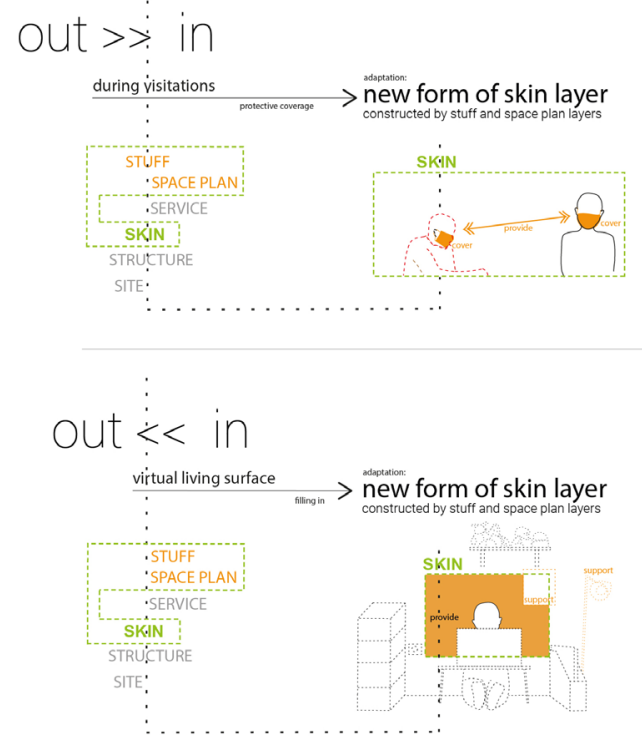

Similar relations between new forms of layer and other layers can be seen in how the virtual interface in devices influence changes to other layers to provide an appropriate background for video conferencing. Examples are the placements of stuff layer (such as books and furniture) in addition to placement of space plan layer (backgrounds, partitions) to create the appropriate background within the virtual interface that is deemed acceptable for view by the public. In this sense, the aesthetic presence of the virtual interface as the skin layer is directly influenced by other kinds of layers.

\section{Reconfiguration of multiple layers together: A prolonged change}

While the other types of layer adaptation discussed in the previous sections assisted with more temporary actions, the reconfiguration of multiple layers together is aimed to help with a more prolonged change that provides continuous protection for dwellers. For example, if family members have COVID-19 symptoms and are required to self-isolate at home, the layers of the domestic environment must be continuously rearranged and redistributed so the patient can be cared for and the others can be protected. All the previous types of layer adaptation are employed altogether to create a more permanent resilient interior. The diagram in Figure 5 demonstrates the reconfiguration of different layers to divide 
or cover space or bodies. The green dashed box groups together multiple layers, indicating they have been reconfigured to provide continuous protection.

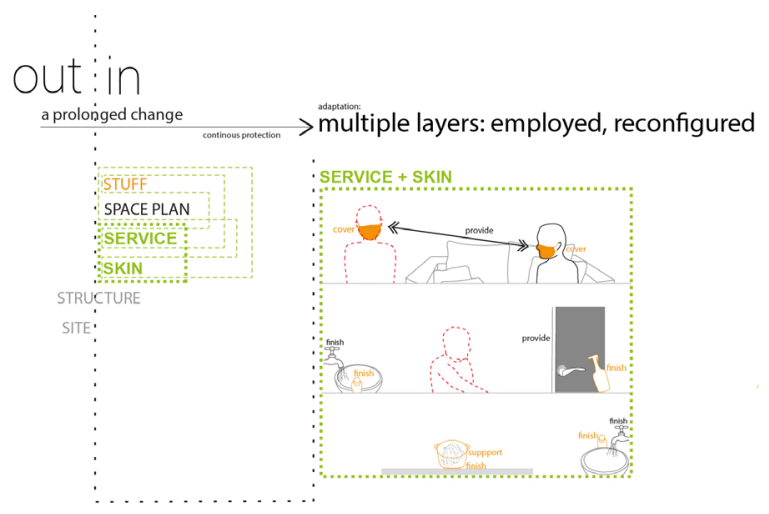

Figure 5

Some layers

interact

through the reconfiguration of multiple layers to be used together (Image by authors)

The layers enable isolation for some family members, dividing dwellers who are healthy from dwellers who have COVID-19. The stuff, space plan, and skin layers are rearranged, thus constructing a new form of skin layer. However, this is achieved not only by covering surfaces but by separating the spaces used for activities and the objects used by different dwellers.

Some layers are also involved in the sanitising process, ensuring no additional transmission of the virus occurs between dwellers. The stuff and service layers are rearranged to create a new service layer, which performs more intensively, incorporating parts of the stuff and service layers. This new service layer is also redistributed to cover all possible spaces where activities of infected and uninfected dwellers must take place. Examples include the division of bathrooms and the creation of multiple hand-washing stations within the home, or the use of stoves to boil clothes and other stuff belonging to the dwellers, demonstrating a more extreme level of precaution within the domestic environment.

\section{The Activity of Domestic Layers to Create a Resilient Interior: Conclusion}

This paper aims to expand Brand's (1995) conceptual proposition of shearing layers as the basis for investigating changes within the domestic environment during the COVID-19 pandemic. The present study highlights how such a framework enables us to recognise different forces and conditions that shape the built environment, thus understanding the built environment's adaptation processes more thoroughly. This study points out that, during the COVID-19 
pandemic, a resilient built environment must respond to more complex threats, resulting in potentially more rapid changes. The use of an interior perspective enables layers to be addressed as instruments with particular spatial qualities that are useful for protecting against such threats as COVID-19. The diagram in Figure 6 proposes an alteration of Brand's diagram, illustrating a shifting understanding of layers' adaptation during the pandemic. The addition of new lines that connect layers represents the interaction between some layers, and the increased quantity of arrows shows the increasing intensity of the layers' adaptations. Lastly, the diagram uses different line colours, which represent the different adaptation principles and performance of such principles within each occurrence of change.

Figure 6 Occurrences of layers' adaptation in the domestic environment to protect against various threats during the COVID-19 pandemic (Image by authors, adapted from Brand (1995))

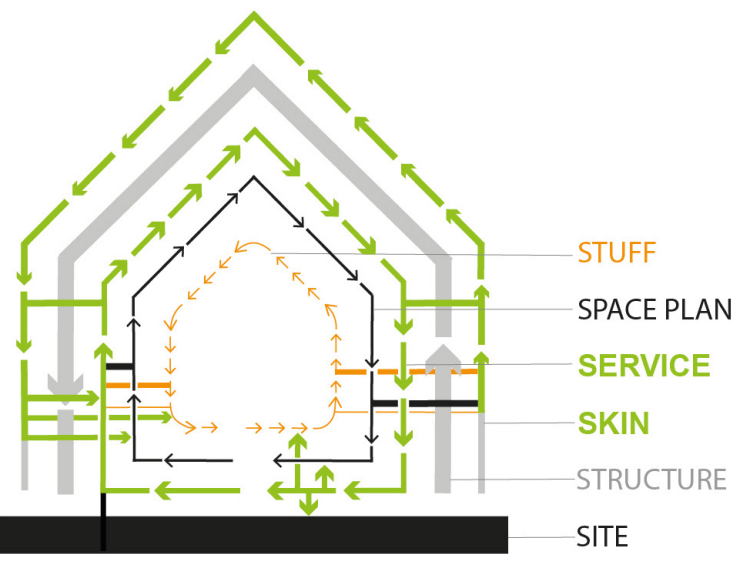

This paper re-reads the understanding of the domestic environment's layers, identifying the layers' adaptation principles and performance of change in a domestic environment during the pandemic. The principles presented in this study significantly alter Brand's understanding of layers and consist of the way layers interact while changing, the emergence of new forms of layers and the repositioning of layers' configurations. The inquiry then highlights how these principles assist with particular activities of layers in response to new needs during the COVID-19 pandemic. Among the occurrences that drive change are the intensified use of each layer, the merging of two layers to assist movements in and outside the home, the construction of a new form for a layer to help apply new procedures and systems or new uses of technology, and the reconfiguration of multiple layers together in the event of more prolonged change. These occurrences demonstrate different rates of layers' change and the different position of each layer in accordance with other layers. Some layers may change more often, 
while some layers may be merged with another layer. This study's reading also places more emphasis on the site layer, of which the inhabitants expand its role, which was largely limited in Brand's proposition.

Current architecture and interior discourses use the concept of layers in their understanding of the built environment. By viewing layers through the interiority perspective as instruments that assist with a particular performance in a space, this paper expands the current understanding of layers. It drives the understanding of change in the domestic environment beyond physical deterioration to include the need for conditions and qualities that ensure the safety of a dwelling's inhabitants. This understanding is important to guide our perspective of a building's adaptation to changing forces in space and time. Further exploration of layers' adaptation in other spatial contexts and events is important to expand the discussion of layers and corresponding knowledge of how buildings attain resilience under unprecedented conditions.

\section{References}

Adams, R., \& Marlor, R. (2019). Breaking the binary oppositions of the interior: A momentary permanence. Interiority, 2(2), 113128. https://doi.org/10.7454/in.v2i2.58

Alexander, C. (1975). The Oregon experiment. New York: Oxford University Press.

Alexander, C. (1979). The timeless way of building. New York: Oxford University Press.

Attiwill, S. (2012). Beyond building: Interior designs. Perth: Curtin University.

Banham, R. (1984). The Architecture of the well-tempered environment (2nd ed). Chicago: University of Chicago Press.

Bloomfield, S. F., Exner, M., Signorelli, C., Nath, K. J., \& Scott, E. A. (2012). The chain of infection transmission in the home and everyday life settings, and the role of hygiene in reducing the risk of infection. International Scientific Forum on Home Hygiene. https://www.ifh-homehygiene.org/review/chaininfection-transmission-home-and-everyday-life-settingsand-role-hygiene-reducing-risk

Brand, S. (1995). How buildings learn: What happens after they're built. New York: Penguin Books. 
de Certeau, M. (1984). The practice of everyday life, (S. Rendall, Trans.). Berkeley: University of California. (Original work published 1974).

Dietz, L., Horve, P. F., Coil, D. A., Fretz, M., Eisen, J. A., \& Van Den Wymelenberg, K. (2020). 2019 Novel coronavirus (COVID-19) pandemic: Built environment considerations to reduce transmission. MSystems, 5(2). https://doi.org/10.1128/ mSystems.00245-20

Duan, S. M., Zhao, X.S., Wen, R.F., Huang, J.J., Pi, G.H., Zhang, S. X., Han, J., Bi, S. L., Ruan, L., Dong, X. P., \& SARS Research Team. (2003). Stability of SARS coronavirus in human specimens and environment and its sensitivity to heating and UV irradiation. Biomedical and Environmental Sciences: BES, 16(3), 246-255. PMID: 14631830

Duffy, F. (1990). Measuring building performance. Facilities, 8(5), 1720. https://doi.org/10.1108/EUM0000000002112

Franck, K. A., \& Lepori, R. B. (2000). Architecture inside out. Chichester: Wiley.

Friedberg, A. (2006). The virtual window: From Alberti to Microsoft. Cambridge: MIT Press.

Handa, R. (2015). Allure of the incomplete, imperfect, and impermanent: Designing and appreciating architecture as nature. New York: Routledge. Taylor \& Francis Group.

Klasto, C. (2019). Tokyo's kyōshō jūtaku: Nature through the inside, outside and the in-between. Interiority, 2(2), 155-176. https://doi.org/10.7454/in.v2i2.63

Leatherbarrow, D. (2002). Uncommon ground:Architecture, technology, and topography. Cambridge: MIT Press.

Leatherbarrow, D., \& Mostafavi, M. (2002). Surface architecture. Cambridge: MIT Press.

Lens, K., \& Van Cleempoel, K. (2015). Monasteries - The layered capture of rhythm in space and time through rituals by 'moulage.' Interiors, 6(3), 289-305. https://doi.org/10.1080/ 20419112.2015.1125626

Marinic, G. (2018). The interior architecture theory reader. New York: Routledge. 
Martinelli, P. M. (2019). House, street, city: Le Corbusier's research towards a new urban interior. Interiority, 2(2), 129-153. https://doi.org/10.7454/in.v2i2.57

Schneider, T., \& Till, J. (2007). Flexible housing. New York: Architectural Press, Routledge.

Till, J. (2013). Architecture depends. Cambridge: MIT Press.

van Doremalen, N., Bushmaker, T., Morris, D. H., Holbrook, M. G., Gamble, A., Williamson, B. N., Tamin, A., Harcourt, J. L., Thornburg, N. J., Gerber, S. I., Lloyd-Smith, J. O., de Wit, E., \& Munster, V. J. (2020). Aerosol and surface stability of SARSCoV-2 as compared with SARS-CoV-1. New England Journal of Medicine, 382(16), 1564-1567. https://doi.org/10.1056/ NEJMc2004973

Wang, Y., Di, Y., Ye, J., \& Wei, W. (2020). Study on the public psychological states and its related factors during the outbreak of coronavirus disease 2019 (COVID-19) in some regions of china. Psychology, Health \& Medicine, 1-10. https://doi.org/10.1080/13548506.2020.1746817

Warakanyaka, A. A. A. S., \& Yatmo, Y. A. (2018). Understanding the importance of time in interior architectural design method. SHS Web of Conferences, 41, 04009. https://doi.org/10.1051/ shsconf/20184104009

Warakanyaka, A. A. A. S., \& Yatmo, Y. A. (2018). Tracing the progression of inhabitation through interior surface in Semarang Old Town. Interiority, 1(1), 64-78. https://doi.org/10.7454/in.v1i1.9

Weinthal, L. (2011). Toward a new interior: An anthology of interior design theory. New York: Princeton Architectural Press.

WHO. (2020). Advice on the use of masks in the context of COVID-19: Interim guidance, 6 April 2020. https://apps.who.int/iris/ handle/10665/331693

Yatmo, Y. A., Atmodiwirjo, P., \& Harahap, M. M. Y. (2020). Hand touches on the surfaces of a healthcare waiting area. Journal of Hospital Infection, 105(2), 383-385. https://doi.org/10.1016/j. jhin.2020.04.042

Yatmo, Y. A., Putra, N., Harahap, M. M. Y., \& Saginatari, D. P. (2018). Evaluation of spatial layout in health care waiting areas based on simulation of droplet movement trace. International Journal of Technology, 9(5), 888-897. https:// doi.org/10.14716/ijtech.v9i5.2106 
